\title{
White Rice Intake Varies in Its Association with Metabolic Markers of Diabetes and Dyslipidemia Across Region among Chinese Adults
}

\author{
Fei Dong $^{\mathrm{a}}$ Annie-Green Howard ${ }^{\mathrm{b}, \mathrm{c}}$ Amy H. Herring ${ }^{\mathrm{b}, \mathrm{c}}$ Barry M. Popkin ${ }^{\mathrm{a}, \mathrm{b}}$ \\ Penny Gordon-Larsen ${ }^{a, b}$ \\ ${ }^{a}$ Department of Nutrition, Gillings School of Global Public Health and School of Medicine at the University of North \\ Carolina at Chapel Hill, ${ }^{b}$ Carolina Population Center, University of North Carolina, ' Department of Biostatistics, \\ Gillings School of Global Public Health, University of North Carolina, Chapel Hill, N.C., USA
}

\section{Key Words}

White rice intake $\cdot$ Diabetes $\cdot$ Dyslipidemia $\cdot$ Chinese adults

\begin{abstract}
Background/Aims: There are inconsistent associations between white rice consumption and diabetes and dyslipidemia, perhaps due to the nature of samples studied and quality of diet data. Methods: Using regionally diverse data from adults enrolled in the China Health and Nutrition Survey $(n=7,878)$ with diet data from three repeated 24-hour recalls and fasting blood to derive diabetes and dyslipidemia, we examined the odds of diabetes and dyslipidemia in participants across region-specific tertiles of percent energy from white rice consumption. Results: The prevalence of undiagnosed diabetes, high triglycerides, high low-density lipoprotein (LDL), low high-density lipoprotein (HDL), and atherogenic dyslipidemia (AD) was 4.7\%, 31.8\%, 31.3\%, $25.9 \%$, and $14.6 \%$, respectively. We found an inverse association between the highest (versus lowest) tertile of rice intake and diabetes in Central China (odds ratio (OR): $0.59,95 \%$ confidence interval ( $\mathrm{Cl}): 0.36-0.99)$. The highest rice consumption was also associated with high triglycerides (OR: 1.46, 95\% Cl: 1.09-1.95), low HDL (OR: 1.38, 95\% Cl: 1.03-1.85), and AD (OR: 1.63, 95\% Cl: 1.15-2.31) in North China, and low LDL
\end{abstract}

(OR: 0.54, 95\% Cl: 0.42-0.69) in Central China. Conclusions: The association between white rice consumption and diabetes and dyslipidemia markers varied across regions of China, suggesting a role of other dietary and health-related exposures, beyond rice.

(c) 2015 S. Karger AG, Basel

\section{Introduction}

The prevalence of type 2 diabetes mellitus (T2DM) in China has increased dramatically from $0.8 \%$ in 1979 to $8 \%$ in 2012 [1], along with a surge in dyslipidemia [2]. T2DM and dyslipidemia often coexist [3]. White rice is the major staple food in Asia, but with a regional variation (ranging from high (Southern China) to low (Central China) consumption). The literature on rice consumption and T2DM is mixed [4-11], ranging from positive [8, $10,11]$, null $[4,5,9]$ to negative [7], as is the association with markers of dyslipidemia (positive or no association with high triglycerides and/or low high-density lipoprotein (HDL) $[5,10,12-15])$. These inconsistent findings may relate to the nature of samples studied, quality of diet data, or different biological effects rather than heterogeneity in results across studies.

\section{KARGER 125}

(c) 2015 S. Karger AG, Base

0250-6807/15/0664-0209\$39.50/0

E-Mail karger@karger.com

www.karger.com/anm
Penny Gordon-Larsen, $\mathrm{PhD}$

University of North Carolina at Chapel Hill

137 East Franklin Street

Campus Box \#8120, Chapel Hill, NC 27514 (USA)

E-Mail pglarsen@unc.edu 
To address this inconsistency, we used regionally diverse data from adults enrolled in the China Health and Nutrition Survey (CHNS) to examine the association between white rice intake and markers of diabetes and dyslipidemia (high triglycerides, high low-density lipoprotein (LDL), low HDL, and atherogenic dyslipidemia (AD, high triglycerides, and low HDL, an important risk factor for cardiovascular disease)), with attention to the regional variation in rice consumption.

\section{Methods}

\section{China Health and Nutrition Survey}

In 7 survey rounds, the CHNS collected health data from 228 communities in 9 diverse provinces throughout China (North: Heilongjiang, Liaoning; Central: Shandong, Henan, Jiangsu; South: Hunan, Hubei, Guangxi, Guizhou) from 1991 to 2009. Questionnaires were used to collect demographic, socioeconomic, anthropometric, behavioral, and health information. The 2009 survey collected fasting blood for the first time. Using a multistage, random cluster design, a stratified probability sample was used to select counties and cities stratified by income using State Statistical Office definitions [16], and then, communities and households were selected from these strata. The CHNS cohort initially mirrored national age-gender-education profiles [17-19] and the provinces in the CHNS sample constituted $44 \%$ of China's population in 2009 (according to 2009 census). Survey procedures have been described elsewhere [20]. The study was approved by the Institutional Review Board at the University of North Carolina at Chapel Hill, the China-Japan Friendship Hospital, Ministry of Health, and the Institute of Nutrition and Food Safety, China Centers for Disease Control, and the subjects gave informed consent for participation.

\section{Analysis Sample}

We restricted analyses to adults ( $\geq 18$ and $<98$ years) at the 2009 exam $(\mathrm{n}=8,616)$. We excluded individuals who were pregnant ( $n=61,0.7 \%)$, or missing diet $(n=115,1.3 \%)$ or blood biomarker ( $n=19,0.2 \%$ ) data, or were not fasting before the blood withdraw$\mathrm{al}(\mathrm{n}=402,4.7 \%)$. We further excluded individuals with previous diabetes diagnosis to reduce the possibility of reverse causality $(\mathrm{n}=$ $232,2.7 \%$ ), resulting in final analytic samples of 7,628 (diabetes) and 7,878 (dyslipidemia). Excluded subjects were younger, slightly better educated, and more likely to be in the Southern region than the analytic sample. There were no statistically significant differences in sex, body mass index (BMI), income, and urbanization levels.

\section{Ascertainment of Diabetes and Dyslipidemia}

Blood samples were collected by venipuncture after overnight fasting (12 ml). Whole blood was immediately centrifuged and serum immediately used to measure glucose using a glucose oxidase phenol 4-aminoantipyrine peroxidase kit (GOD-PAP; Randox, Crumlin, UK). Diabetes was classified using fasting blood glucose $(\mathrm{FBG}) \geq 7.0 \mathrm{mmol} / \mathrm{l}(\mathrm{n}=359)$ in our main analysis [21]. In the sensitivity analysis, we defined diabetes using hemoglobin $\mathrm{A} 1 \mathrm{c} \geq 6.5 \%$ $(\mathrm{n}=447)[22]$. Total triglycerides, HDL, and LDL were measured using the glycerol-phosphate oxidase method and the polyethylene glycol (PEG)-modified enzyme method by determiner regents (Kyowa Medex Ltd., Tokyo, Japan), using a Hitachi 7600 automated analyzer (Hitachi Inc., Tokyo, Japan). Dyslipidemia was defined following the International Diabetes Federation criteria (high triglycerides: $\geq 150 \mathrm{mg} / \mathrm{dl}, \mathrm{n}=2,488$; low HDL: men $<40 \mathrm{mg} /$ $\mathrm{dl}$, women $<50 \mathrm{mg} / \mathrm{dl}, \mathrm{n}=2,450$; high LDL: $>130 \mathrm{mg} / \mathrm{dl}, \mathrm{n}=2,041$ ) or use of a lipid lowering medication $(n=31)$ [23]. AD was defined as having high triglycerides and low $\operatorname{HDL}(\mathrm{n}=1,148)$ [24].

\section{Dietary Assessment}

The dietary intake was assessed using 3 consecutive 24-hour recalls at the individual level and a food inventory at the household level collected during the same 3-day period, randomly starting from Monday to Sunday. All foods available in the household were measured on a daily basis for the food inventory. For the 24-hour recalls, trained interviewers recorded types and amounts of foods consumed. Daily average consumption (g/day) of white rice and other foods were estimated. The proportion of energy from rice was then calculated by dividing the daily average energy intake from rice by the daily average total energy intake. The nutrient contents were based on a Chinese food composition table [25]. Raw rice was reported, but mostly consumed as steamed rice. The total energy intake was validated with the doubly labeled water method in the Human Nutrition Research Center, Tufts University (correlation coefficients between the two methods were 0.56 for men and 0.60 for women [26]).

\section{Statistical Analyses}

White rice intake (\% total energy) was categorized into regionspecific tertiles (North, Central, South). We tested differences in the descriptive characteristics by categories of rice consumption and by region using ANOVA (continuous variables) and Chisquare tests (categorical variables). We tested correlations between rice consumption and other food groups (percent energy from food groups) using Pearson's correlation and generated two-factor analysis-derived dietary patterns (online suppl. methods; for all online suppl. material, see www.karger.com/ doi/10.1159/000430504) to capture the dietary confounders as dietary pattern scores [27]. We used multivariable logistic regression models to estimate odds ratios (OR), adjusting for household clustering using the robust cluster command. Given a statistically significant $(\mathrm{p}<0.01)$ interaction for rice by region and because of substantial regional variation in diet, cooking methods, and lifestyle factors across China, we ran models by the region.

We present results for multivariable fully adjusted models, including age (continuous with linear and quadratic terms), gender, education (below/equal to/above high school), urbanicity (low/ medium/high), hypertension diagnosis (yes/no), total physical activity (METs/week, quartiles), total energy intake (kcal/day, quartiles), dietary pattern scores (quartiles), and BMI $\left(\mathrm{kg} / \mathrm{m}^{2}\right.$, quartiles). In addition, diabetes models included fiber ( $\mathrm{g} /$ day, quartiles) and magnesium (mg/day, quartiles) intakes and the dyslipidemia models included fat intake (\% energy from fat, quartiles). We tested smoking (yes/no), alcohol intake (g/day, quartiles), and income (low/medium/high), but they did not change the estimates and, thus, were not retained; neither were the fat intake and lipid levels in the diabetes models. We also tested for the interaction between rice intake and age and gender in all models, and with dyslipidemia medication use in the dyslipidemia 
models. Tests for linear trend across increasing rice intake categories were conducted by modeling the categories as a continuous variable using the median value for each category. Since white rice and wheat products are the two main types of staple foods consumed in China, we also evaluated the association of 'substituting' $10 \%$ of total energy from wheat products as staple food (wheat bun and noodles) for $10 \%$ of total energy from rice with diabetes and dyslipidemia. We did the analysis by simultaneously including rice and wheat products intake as continuous variables in the same multivariable model. The difference in their coefficients and covariance was used to estimate the OR and 95\% confidence intervals (CI) for the substitution, respectively [28]. All analyses were performed using Stata 13 (StataCorp), with two-sided $\mathrm{p}<$ 0.05 considered statistically significant.

\section{Results}

Median percent energy from white rice was the highest in the South (43\%) and lowest in the Central (13.5\%) region. Overall, a greater proportion of participants at the highest (versus lowest) rice consumption tertile were leaner, of lower education and income, and living in lessurbanized areas (table 1). The physical activity was higher, whereas fiber, fat, and magnesium intakes were lower at the highest rice consumption tertile. Most descriptive characteristics varied significantly $(\mathrm{p}<0.001)$ across regions.

Correlations between consumption of rice and other food groups are shown in online supplementary table 1. Rice consumption was negatively correlated with wheat products consumption. To control for dietary confounding, we used region-specific factor scores to examine other dietary factors (beyond rice) with a potential influence on diabetes and dyslipidemia markers (factor loadings in table 2). Two Northern patterns were characterized by high consumption of low-sugar wheat and milk (western pattern), and high cakes, pastries, and soymilk (traditional pattern), respectively. In the Central region, we identified a low-wheat pattern (low wheat as staple food and high fish and seafood) and a western pattern (high low-sugar wheat, red meat, eggs, and milk). Two Southern patterns were featured by a high intake of cakes, pastries, and milk (western pattern) and high wheat as staple food (high-wheat pattern), respectively. White rice consumption had a strong positive correlation with the lowwheat pattern in the Central region $(\mathrm{r}=0.64, \mathrm{p}<0.05)$, moderate negative correlations with the traditional pattern in the North, western pattern in the Central region, and both western and high-wheat patterns in the Southern region $(\mathrm{r}=-0.24,-0.22,-0.27$, and -0.29 , respectively, $\mathrm{p}<0.05)$.

Rice, Diabetes, and Dyslipidemia in China

\section{Diabetes}

In 2009, 359 out of 7,628 participants had undiagnosed diabetes, with a variation across regions (North: 5.2\%, Central: $5.7 \%$, South: 3.7\%; table 3). Fully adjusted multivariable models suggest that the highest (versus the lowest) category of rice intake was associated with lower prevalence of diabetes in the Central region (OR: 0.59, 95\% CI: 0.36-0.99). In online supplementary table 2, we present results for crude age- and gender-adjusted models and multivariable models without BMI adjustment. We did not detect the interaction between rice intake and age or gender.

\section{Dyslipidemia}

The prevalence of high triglycerides, high LDL, low $\mathrm{HDL}$, and $\mathrm{AD}$ varied across regions (table 4 ). Comparing the highest rice consumption category to the reference, there were significantly higher odds of high triglycerides (OR: 1.46, 95\% CI: 1.09-1.95), low HDL (OR: 1.38, 95\% CI: $1.03-1.85$ ), and AD (OR: 1.63, 95\% CI: 1.15-2.31) in the Northern region, and lower odds of high LDL (OR: 0.54, 95\% CI: 0.42-0.69) in the Central region. In online supplementary table 3 , we present results for crude ageand gender-adjusted models and multivariable models without BMI adjustment. We did not detect an interaction between rice intake and age, gender, or dyslipidemia medication.

\section{Sensitivity Analyses}

Using hemoglobin Alc to define diabetes did not considerably change the results compared to using the FBG (online suppl. table 4). Substitution analysis showed that the replacement of $10 \%$ energy from wheat products with $10 \%$ energy from rice was associated with $12 \%$ lower odds of diabetes (95\% CI: $0.3-22 \%)$ and $12 \%$ lower odds of high LDL ((95\% CI: 7-18\%) in the Central region (online suppl. fig. 1) and $16 \%$ higher odds of high triglycerides (95\% CI: 5-27\%), 11\% higher odds of low HDL (95\% CI: $0.2-23 \%)$, and $13 \%$ higher odds of $\mathrm{AD}$ (95\% CI: $1-26 \%)$ in the North.

\section{Discussion}

In this population-based cross-sectional study of Chinese adults, we capitalized on well-measured diet intake data from three 24-hour recalls to examine how white rice consumption was associated with diabetes and dyslipidemia, taking other dietary factors into account. A higher rice consumption was associated with a lower 


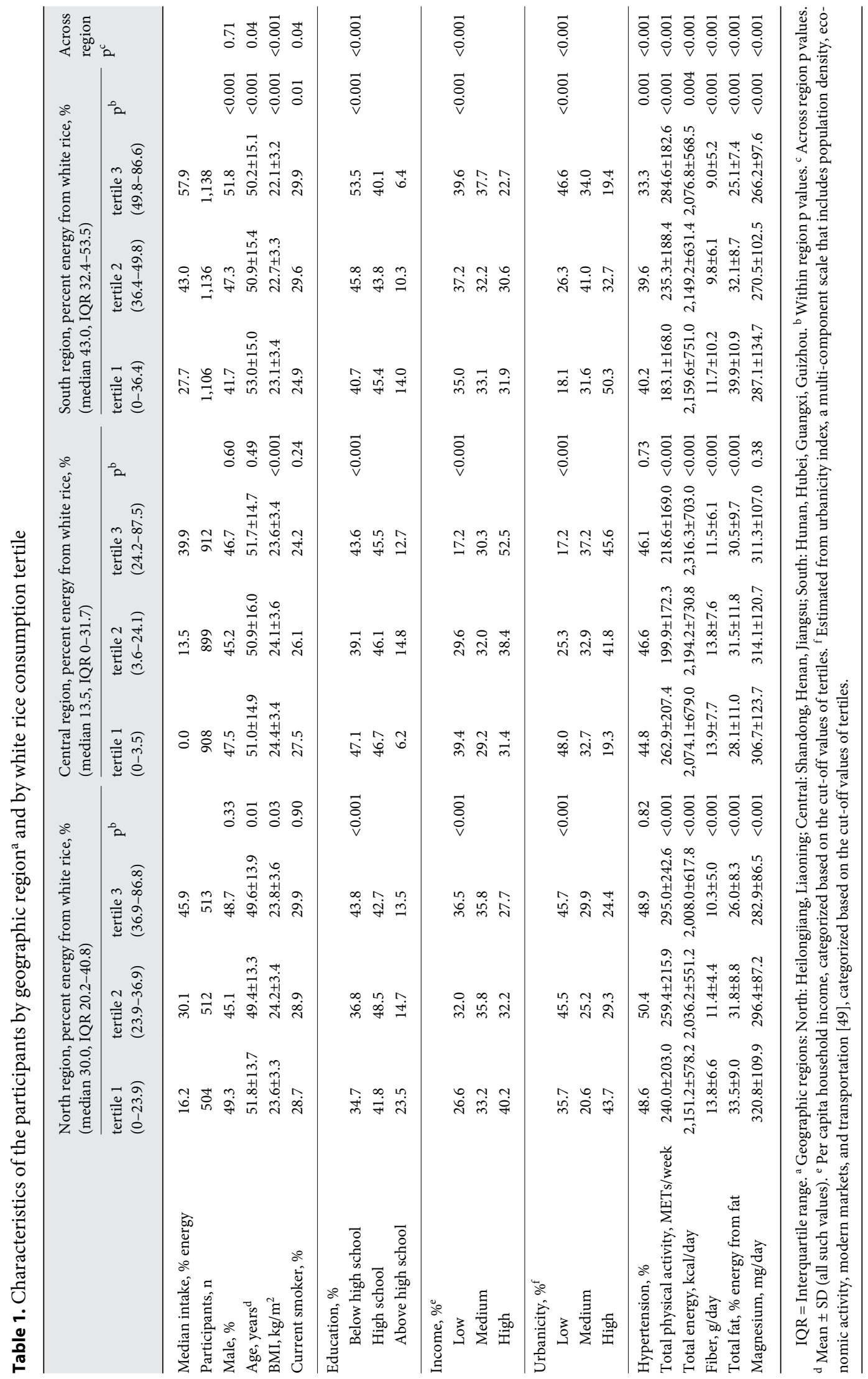


Table 2. Factor loadings for two dietary patterns in each geographic region ${ }^{\text {a }}$ derived from principal factor analysis and Pearson's correlation coefficients between pattern scores and food/nutrients intake

\begin{tabular}{|c|c|c|c|c|c|c|}
\hline & \multicolumn{2}{|c|}{ North region } & \multicolumn{2}{|c|}{ Central region } & \multicolumn{2}{|c|}{ South region } \\
\hline \multicolumn{7}{|l|}{ Food groups, g/day } \\
\hline Wheat products as staple food ${ }^{b}$ & $-0.27^{\mathrm{e}}$ & 0.18 & -0.77 & 0.02 & -0.05 & 0.52 \\
\hline Low-sugar wheat products ${ }^{c}$ & 0.64 & 0.00 & 0.05 & 0.36 & 0.47 & -0.20 \\
\hline Starchy tubers and starch & -0.43 & -0.19 & -0.25 & 0.04 & 0.07 & 0.28 \\
\hline Fruits and vegetables & -0.13 & 0.12 & 0.26 & 0.14 & 0.22 & 0.15 \\
\hline Red meat & 0.23 & 0.33 & 0.43 & 0.38 & 0.28 & -0.14 \\
\hline Poultry & 0.35 & -0.08 & 0.41 & 0.30 & 0.28 & -0.37 \\
\hline Fish and seafood & 0.29 & 0.35 & 0.66 & 0.18 & 0.37 & -0.06 \\
\hline Dried legumes & 0.31 & 0.12 & 0.27 & 0.09 & 0.08 & 0.33 \\
\hline \multicolumn{7}{|l|}{ Correlation coefficients } \\
\hline White rice, $\%$ energy & $-0.07^{*}$ & $-0.24^{*}$ & $0.64^{*}$ & $-0.22^{*}$ & $-0.27^{*}$ & $-0.29^{*}$ \\
\hline Total energy, kcal/day & -0.03 & $0.27^{*}$ & 0.03 & $0.28^{*}$ & $0.16^{*}$ & $0.13^{*}$ \\
\hline Total fat, \% energy & $0.23^{*}$ & $0.24^{*}$ & $0.34^{*}$ & $0.23^{*}$ & $0.22 *$ & $-0.09^{*}$ \\
\hline
\end{tabular}

${ }^{a}$ Geographic regions: North: Heilongjiang, Liaoning; Central: Shandong, Henan, Jiangsu; South: Hunan, Hubei, Guangxi, Guizhou. ${ }^{b}$ Noodles, wheat buns etc. ${ }^{\mathrm{c}}$ Biscuits, bread, dumplings. ${ }^{\mathrm{d}}$ Corn and millet. ${ }^{\text {e }}$ Factor loadings $\geq 0.20$ are bold. ${ }^{*} \mathrm{p}<0.05$.

Table 3. ORs (95\% CIs) of diabetes according to categories of percent energy from white rice and geographic region ${ }^{\mathrm{a}}$

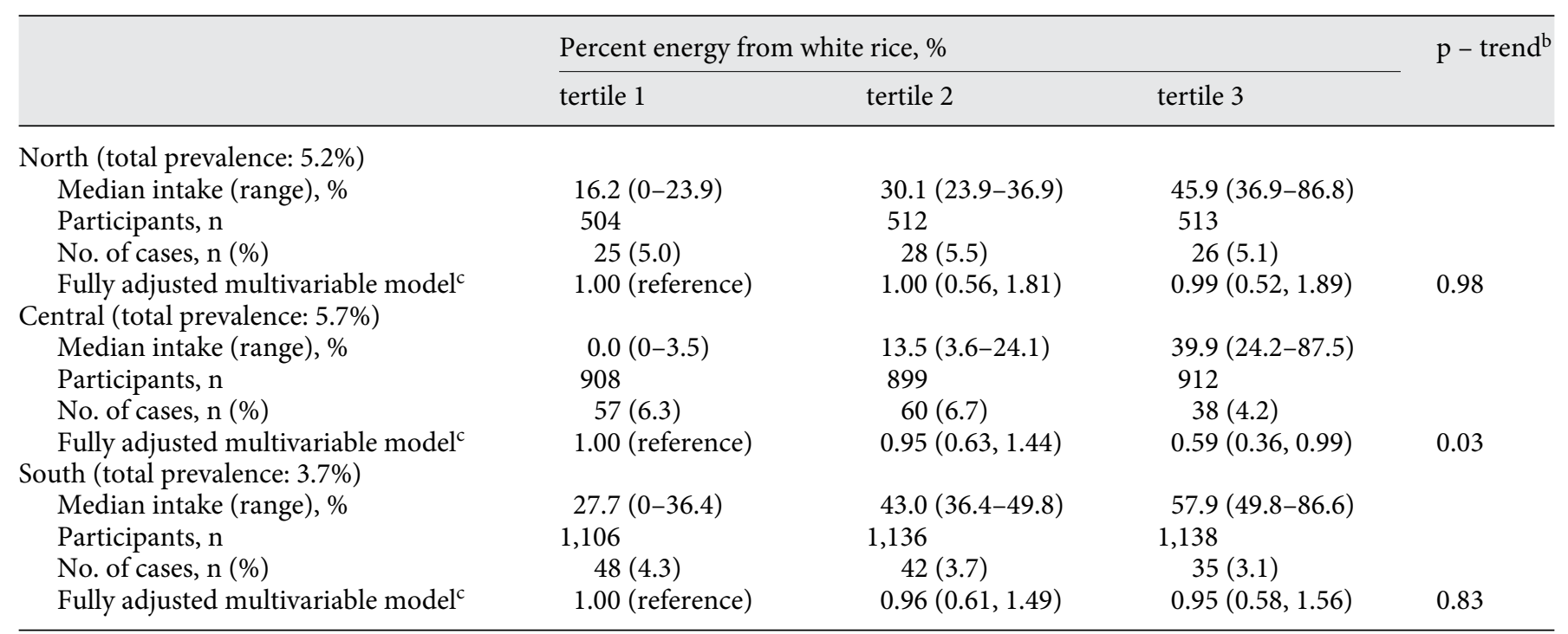

Diabetes was defined as a fasting blood glucose measurement $\geq 7.0 \mathrm{mmol} / \mathrm{l} .{ }^{\text {a }}$ Geographic regions: North: Heilongjiang, Liaoning; Central: Shandong, Henan, Jiangsu; South: Hunan, Hubei, Guangxi, Guizhou. ${ }^{\mathrm{b}}$ Median values were assigned to each category and the variable was modeled as continuous to test linear trend. ${ }^{c}$ Adjusted for age (continuous with linear and quadratic terms), gender, education (below high school/high school/above high school), urbanicity (low/medium/high), hypertension diagnosis (yes/no), total physical activity (METs/week, quartiles), total energy intake (kcal/day, quartiles), fiber intake (g/day, quartiles), magnesium intake (mg/day, quartiles), dietary pattern scores (quartiles), and BMI ( $\mathrm{kg} / \mathrm{m}^{2}$, quartiles). 
Table 4. ORs (95\% CIs) of dyslipidemia markers according to categories of percent energy from white rice and geographic region ${ }^{\mathrm{a}}$

Percent energy from white rice, $\%$

$\mathrm{p}-$ trend $^{\mathrm{b}}$

tertile $1 \quad$ tertile 2 tertile 3

High triglycerides

North (total prevalence: $37.2 \%$ )

Median intake (range), \%

Participants, $\mathrm{n}$

No. of cases, \%

533

$182(34.2)$

1.00 (reference)

tertile 2

tertile 3

Fully adjusted multivariable model ${ }^{\mathrm{c}}$

Central (total prevalence: $29.5 \%$ )

Median intake (range), \%

Participants, $\mathrm{n}$

$0.0(0-3.5)$

942

No. of cases, \%

278 (29.5)

1.00 (reference)

$30.1(23.9-36.9)$
532
$204(38.4)$
$1.19(0.90,1.56)$
$13.5(3.6-24.1)$
941
$284(30.2)$
$1.04(0.83,1.30)$
$43.0(36.4-49.8)$
1,152
$357(30.7)$
$0.87(0.71,1.05)$

$45.9(36.9-86.8)$

532

$208(39.1)$

$1.46(1.09,1.95)$

0.01

Fully adjusted multivariable model ${ }^{\mathrm{c}}$

$27.7(0-36.4)$

1,153

Median intake (range), $\%$

Participants, $\mathrm{n}$

407 (35.3)

$0.87(0.71,1.05)$

$39.9(24.2-87.5)$

941

$271(28.8)$

$1.00(0.79,1.24)$

0.97

Fully adjusted multivariable model ${ }^{c}$

1.00 (reference)

$57.9(49.8-86.6)$

1,152

$318(27.6)$

$0.87(0.69,1.11)$

0.24

High LDL

North (total prevalence: $30.8 \%$ )

No. of cases, \%

Fully adjusted multivariable model ${ }^{\mathrm{c}}$

$175(32.8)$

1.00 (reference)

348 (36.9)

1.00 (reference)

Fully adjusted multivariable model ${ }^{\mathrm{c}}$

South (total prevalence: $30.4 \%$ )

No. of cases, $\%$

Fully adjusted multivariable model ${ }^{c}$
$352(30.5)$

1.00 (reference)
$150(28.2)$
$0.86(0.65,1.16)$

$325(34.5)$

$0.78(0.63,0.97)$

367 (31.9)

$1.20(0.99,1.45)$
$166(31.2)$

$1.18(0.87,1.60)$

0.28

$251(26.7)$

$0.54(0.42,0.69)$

$<0.001$

$331(28.7)$

$1.21(0.94,1.56)$

0.12

\section{Low HDL}

North (total prevalence: $31.3 \%$ )

No. of cases, $\%$

Fully adjusted multivariable model ${ }^{\mathrm{c}}$

Central (total prevalence: $26.3 \%$ )

No. of cases, $\%$

Fully adjusted multivariable model ${ }^{\mathrm{c}}$

South (total prevalence: $23.1 \%$ )

No. of cases, $\%$

Fully adjusted multivariable model ${ }^{\mathrm{c}}$

Atherogenic dyslipidemia

North (total prevalence: 19.5\%)

No. of cases, $\%$

Fully adjusted multivariable model ${ }^{\mathrm{c}}$

Central (total prevalence: 14.0\%)

No. of cases, \%

Fully adjusted multivariable model ${ }^{\mathrm{c}}$

South (total prevalence: $12.8 \%$ )

No. of cases, $\%$

Fully adjusted multivariable model ${ }^{\mathrm{c}}$

$\begin{array}{lc}147(27.6) & 176(33.1) \\ 1.00 \text { (reference) } & 1.20(0.91,1.58) \\ & \\ 244(25.9) & 240(25.5) \\ 1.00 \text { (reference) } & 0.96(0.75,1.22) \\ 282(24.5) & 254(22.1) \\ 1.00 \text { (reference) } & 0.99(0.79,1.25)\end{array}$

$111(20.9)$

$1.30(0.93,1.81)$

$86(16.1)$
1.00 (reference)
133 (14.1)
1.00 (reference)
173 (15.0)
1.00 (reference)

$137(11.9)$

$0.89(0.68,1.16)$
$176(33.1)$

$1.38(1.03,1.85)$

0.03

$258(27.4)$

$1.11(0.86,1.43)$

0.32

$264(22.9)$

$1.21(0.92,1.59)$

0.21

$114(21.4)$

$1.63(1.15,2.31)$

0.01

$133(14.1)$

0.66

$133(11.6)$

$1.05(0.76,1.46)$
$1.04(0.76,1.43)$

High triglycerides: $\geq 150 \mathrm{mg} / \mathrm{dl}$ or taking lipid lowering medication; high LDL: $>130 \mathrm{mg} / \mathrm{dl}$ or taking lipid lowering medication; low

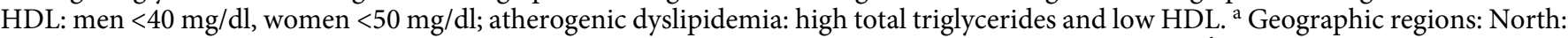
Heilongjiang, Liaoning; Central: Shandong, Henan, Jiangsu; South: Hunan, Hubei, Guangxi, Guizhou. ${ }^{\mathrm{b}}$ Median values were assigned to each category and the variable was modeled as continuous to test linear trend. ${ }^{c}$ Adjusted for age (continuous with linear and quadratic terms), gender, education (below high school/high school/above high school), urbanicity (low/medium/high), hypertension diagnosis (yes/no), total physical activity (METs/week, quartiles), total energy intake (kcal/day, quartiles), fat intake (\% of total energy, quartiles), dietary pattern scores (quartiles), and BMI ( $\mathrm{kg} / \mathrm{m}^{2}$, quartiles). 
prevalence of diabetes and high LDL in the Central region, and higher prevalence of high triglycerides, low HDL, and AD in the North. Substituting the same amount of energy from wheat products with rice showed consistent associations. To our knowledge, this is the first study to examine rice consumption, diabetes, and dyslipidemia associations in three geographic regions of China that vary in consumption of rice, accounting for other dietary factors.

\section{White Rice Intake and Diabetes}

White rice is the staple food in many Asian countries [8]. The association between rice intake and diabetes has been inconsistent in the Asian countries [8-11, 14]. Studies in China, Japan, and Singapore found white rice consumption positively associated with FBG or T2DM $[8,10$, $11,14]$, whereas no association was observed in a Hong Kong population [9]. Particularly, a prospective study conducted in Shanghai, China, reported a T2DM relative risk of 1.78 (95\% CI: 1.48-2.15) comparing middle-aged women who consumed $\geq 300 \mathrm{~g} /$ day (versus $<200 \mathrm{~g} /$ day) of white rice [8]. However, the southern city of Shanghai generally has high rice consumption with less variability relative to the other regions. Moreover, Villegas et al. [8] did not control for other dietary components, which could be important confounders of the rice and diabetes association. Furthermore, Villegas et al. conducted their study in a less-varied geographic area when compared to the place of our study and they relied on self-reported diabetes cases, which likely underestimates true prevalence, given our observed $61 \%$ undiagnosed diabetes. Our use of FBG measures and detailed diet behavior data to control for important dietary confounders improves upon the current literature. While another study in a single province (Jiangsu China) observed a significantly positive association between white rice intake and hyperglycemia risk [14], we found results in the opposite direction, although we have the Jiangsu province in our CHNS Central region. The inconsistency could be partially due to their high loss to follow-up rate (above 40\%) and the potential confounding effect of other dietary components, which were not addressed in their study [14]. In addition, positive associations were observed only among women in Japan and Shanghai, China $[8,11]$. In our study, we tested the effect measure modification by gender, but detected none.

White rice consumption is considerably lower in the Western countries than Asian countries [29]. Studies that found mixed associations have largely been based in the Western countries. No association between diabetes and white rice or refined grains was observed in Australia, Sweden, Finland, or the United States [4, 29-31], whereas another US study found a positive association [29]. Conversely, Soriguer et al. [7] found a negative association between white rice consumption and 6-year-incidence of T2DM in southern Spain. Similarly, a study among US women observed an inverse association between refined grains and T2DM [32]. The inconsistency among studies could be partly explained by differential consumption levels among diverse study populations.

The mechanism of the observed association between white rice intake and diabetes remains uncertain. In our study, rice consumption was positively associated with magnesium intake, which is known to be associated with lower T2DM risk [33]. Nevertheless, the inverse rice and T2DM association remained significant after the magnesium adjustment. We also observed that higher rice consumers had higher levels of physical activity, were more likely to live in less-urbanized areas, and thus were similar in lifestyle to the early stages of the nutrition transition, a period when diabetes prevalence was lower than it is now [34]. In this sense, the lower diabetes prevalence among higher rice consumers observed in our study mimics the condition in China 20 years ago. Although we adjusted for urbanization level and physical activity, it is possible that other unmeasured factors associated with the social and nutrition transition might confound the association.

In our analyses, the inverse association between rice consumption and diabetes in the Central region was slightly attenuated comparing the fully-adjusted model to the multivariable model without the BMI adjustment, indicating that the association could be partly due to the negative relationship between rice intake and BMI in this region, which is consistent with other studies $[11,14,35]$. These findings suggest that a high consumption of white rice might be linked to more healthy diets. In our study, the high rice consumption was strongly correlated with a healthy low-wheat diet pattern (low wheat, high fish and seafood intake) in the Central region. Another possible reason could be the upregulation of lipolysis and the downregulation of lipogenesis by rice protein, which has been reported in a study with rats that showed an improved adiposity and body weight after rice protein feeding [36]. In addition, fried rice is commonly consumed in China by cooling steamed rice in the refrigerator overnight and stir-frying it with oil; recent studies have shown that these processes promote rice starch retrogradation, increase the resistant starch concentration of rice, and reduce the rice calories [37]. However, the inverse asso- 
ciation remained significant after the BMI and diet pattern adjustment, suggesting an independent relationship.

\section{White Rice Intake and Dyslipidemia}

The positive association between high triglycerides and low HDL with white rice consumption in the Northern region confirms some previous studies [12-15, 38], whereas others found no association $[5,10]$. Possible explanations for our findings could be an enhanced hepatic synthesis of VLDL [39] or a decreased clearance of apoBcontaining particles related to a reduced lipoprotein lipase activity induced by a high carbohydrate intake [40, 41]. We also examined the relation between rice consumption and $\mathrm{AD}$, an important risk factor for cardiovascular disease [24], and found a positive association only in the Northern region. In addition, we observed a negative relationship between rice intake and high LDL prevalence in the Central region. Few studies have examined this association. One such study found no association [5], whereas an inverse relationship between the total carbohydrate intake and high LDL has been reported [42]. Another study found mixed results, depending on whether high carbohydrate intake replaced the saturated or polyunsaturated fat intake [43]. The association between rice consumption and LDL remains uncertain and is possibly affected by other dietary components.

It is unclear why associations between rice, diabetes, and dyslipidemia varied across the three regions. Studies have shown a higher prevalence of metabolic syndrome in the North than South regions of China $[44,45]$. The inconsistency may be due to multiple factors, including differences in dietary patterns, cooking methods, and other lifestyle aspects, among residents in these three regions.

Our study is not without limitations. First, the crosssectional study design makes it difficult to establish temporality of rice consumption, diabetes, and dyslipidemia. Second, we were not able to distinguish type 1 from type 2 diabetes, but type 1 diabetes is rare in China [46]. Third, we adjusted for dietary pattern scores to control for dietary confounders. However, due to the complex correlation between foods consumed, this approach cannot completely remove the dietary confounding. As in Rebello et al. [28], we conducted the substitution analysis in sensitivity analysis replacing wheat products with rice to provide additional insights regarding this issue. Fourth, the Chinese food composition table used did not include brown rice, thus we were unable to compare findings for white versus brown rice in relation to diabetes and dyslipidemia. However, brown rice is rarely consumed in China [47], and a randomized controlled trial replacing white rice with brown rice showed no effect on the FBG levels among a Chinese population with diabetes or prediabetes [48].

Strengths of our study included a population-based study sample from the national survey, allowing us to detect differences of the association across three main regions in China, and the use of a detailed three 24-hour dietary recalls to quantify the rice intake and adjust for dietary confounders. In addition, we used biomarkers derived from fasting blood to identify diabetes and dyslipidemia cases, which is critical given a high prevalence of undiagnosed disease in this population.

In summary, our data suggested that a diet high in white rice was associated with a lower prevalence of diabetes and high LDL, and a higher prevalence of high triglycerides, low $\mathrm{HDL}$, and $\mathrm{AD}$ in certain regions of China. Given that white rice is the staple food and main contributor to total energy intake in many parts of China, the role of white rice consumption in relation to diabetes and dyslipidemia is worth further exploration.

\section{Acknowledgments}

The authors thank the National Institute of Nutrition and Food Safety, Chinese Center for Disease Control and Prevention, the Carolina Population Center, the UNC-CH, and all the people involved in the CHNS. This work was supported by the National Institutes of Health, National Heart, Lung, Blood Institute (R01HL108427). This research uses data from the CHNS, funded by the Eunice Kennedy Shriver National Institute of Child Health and Human Development (NICHD) (R01-HD30880), although no direct support was received from the grant for this analysis. We are also grateful to the Carolina Population Center (R24 HD050924) for general support. F.D. is funded by the Fogarty International Center - NIH (D43 TW009077). The funders had no role in the design and analysis of the study or in the writing of this article.

All authors contributed to conception, design, and interpretation of data, F.D. contributed to data analysis, B.M.P. and P.G.L. contributed to the acquisition of data, F.D. and P.G.L. drafted the article and A.G.H., A.H.H., and B.M.P. contributed to critical revision of the article. F.D. and P.G.L. had full access to all the data in the study and take responsibility for the integrity of the data and the accuracy of the data analysis. All authors have read and approved the final article.

\section{Disclosure Statement}

The authors have no conflicts of interest to disclose. 


\section{References}

1 Li MZ, Su L, Liang BY, Tan JJ, Chen Q, Long JX, Xie JJ, Wu Gl, Yan Y, Guo XJ, Gu L: Trends in prevalence, awareness, treatment, and control of diabetes mellitus in mainland china from 1979 to 2012. Int J Endocrinol 2013;2013:753150.

2 Gu D, Gupta A, Muntner P, Hu S, Duan X, Chen J, Reynolds RF, Whelton PK, He J: Prevalence of cardiovascular disease risk factor clustering among the adult population of China: results from the international collaborative study of cardiovascular disease in Asia (InterAsia). Circulation 2005;112:658-665.

3 Schmidt MI, Watson RL, Duncan BB, Metcalf P, Brancati FL, Sharrett AR, Davis CE, Heiss G: Clustering of dyslipidemia, hyperuricemia, diabetes, and hypertension and its association with fasting insulin and central and overall obesity in a general population. Atherosclerosis risk in communities study investigators. Metabolism 1996;45:699-706.

4 Hodge AM, English DR, O’Dea K, Giles GG: Glycemic index and dietary fiber and the risk of type 2 diabetes. Diabetes Care 2004;27: 2701-2706.

5 Khosravi-Boroujeni H, Sarrafzadegan N, Mohammadifard N, Sajjadi F, Maghroun M, Asgari S, Rafieian-kopaei M, Azadbakht L: White rice consumption and CVD risk factors among Iranian population. J Health Popul Nutr 2013;31:252-261.

6 Nanri A, Mizoue T, Yoshida D, Takahashi R, Takayanagi R: Dietary patterns and A1C in Japanese men and women. Diabetes Care 2008;31:1568-1573.

7 Soriguer F, Colomo N, Olveira G, GarcíaFuentes E, Esteva I, Ruiz de Adana MS, Morcillo S, Porras N, Valdés S, Rojo-Martínez G: White rice consumption and risk of type 2 diabetes. Clin Nutr 2013;32:481-484.

8 Villegas R, Liu S, Gao YT, Yang G, Li H, Zheng W, Shu XO: Prospective study of dietary carbohydrates, glycemic index, glycemic load, and incidence of type 2 diabetes mellitus in middle-aged Chinese women. Arch Intern Med 2007;167:2310-2316.

$9 \mathrm{Yu} \mathrm{R}$, Woo J, Chan R, Sham A, Ho S, Tso A, Cheung B, Lam TH, Lam K: Relationship between dietary intake and the development of type 2 diabetes in a chinese population: the Hong Kong dietary survey. Public Health Nutr 2011;14:1133-1141.

10 Zuñiga YL, Rebello SA, Oi PL, Zheng H, Lee J, Tai ES, Van Dam RM: Rice and noodle consumption is associated with insulin resistance and hyperglycaemia in an Asian population. Br J Nutr 2014;111:1118-1128.

11 Nanri A, Mizoue T, Noda M, Takahashi Y, Kato $M$, Inoue $M$, Tsugane S: Rice intake and type 2 diabetes in Japanese men and women: the Japan public health center-based prospective study. Am J Clin Nutr 2010;92:14681477.

12 Mattei J, Hu FB, Campos $\mathrm{H}$ : A higher ratio of beans to white rice is associated with lower cardiometabolic risk factors in Costa Rican adults. Am J Clin Nutr 2011;94:869-876.

13 Song SJ, Lee JE, Paik HY, Park MS, Song YJ: Dietary patterns based on carbohydrate nutrition are associated with the risk for diabetes and dyslipidemia. Nutr Res Pract 2012;6:349356.

14 Shi Z, Taylor AW, Hu G, Gill T, Wittert GA: Rice intake, weight change and risk of the metabolic syndrome development among Chinese adults: the Jiangsu nutrition study (JIN). Asia Pac J Clin Nutr 2012;21:35-43.

15 Song S, Lee JE, Song WO, Paik HY, Song Y: Carbohydrate intake and refined-grain consumption are associated with metabolic syndrome in the Korean adult population. J Acad Nutr Diet 2014;114:54-62.

16 National Bureau of Statistics of China.

17 Wang L: Summary Report of China Nutrition and Health Survey, 2002. Beijing, People's Medical Publishing House, 2002.

18 Ge K: The Dietary and Nutritional Status of Chinese Population (1992 National Nutrition Survey). Beijing, People's Medical Publishing House, 1995, vol. 1.

19 Chen CM: Nutrition status of the Chinese people. Biomed Environ Sci 1996;9:81-92.

20 Popkin BM: The implications of the nutrition transition for obesity in the developing world; in Obesity Epidemiology. Aetiology to Public Health, 2010, p 136.

21 World Health Organization: Definition and Diagnosis of Diabetes Mellitus and Intermediate Hyperglycemia: Report of a WHO/IDF Consultation. Geneva, World Health Organization, 2006, pp 1-50.

22 World Health Organization: Use of Glycated Haemoglobin (HbAlc) in the Diagnosis of Diabetes Mellitus: Abbreviated Report of a WHO Consultation, 2011.

23 Alberti KG, Zimmet P, Shaw J: Metabolic syndrome - a new world-wide definition. A consensus statement from the international diabetes federation. Diabet Med 2006;23:469480.

24 Musunuru K: Atherogenic dyslipidemia: cardiovascular risk and dietary intervention. Lipids 2010;45:907-914.

25 Yang Y, Wang G, Pan X: China Food Composition 2002. Beijing, Peking University Medical Press, 2002, p 136.

26 Yao M, Lichtenstein AH, Roberts SB, Ma G, Gao S, Tucker KL, McCrory MA: Relative influence of diet and physical activity on cardiovascular risk factors in urban Chinese adults. Int J Obes Relat Metab Disord 2003;27:920932.

27 Hu FB: Dietary pattern analysis: a new direction in nutritional epidemiology. Curr Opin Lipidol 2002;13:3-9.

28 Rebello SA, Koh H, Chen C, Naidoo N, Odegaard AO, Koh WP, Butler LM, Yuan JM, van Dam RM: Amount, type, and sources of carbohydrates in relation to ischemic heart disease mortality in a Chinese population: a pro- spective cohort study. Am J Clin Nutr 2014; 100:53-64.

29 Sun Q, Spiegelman D, van Dam RM, Holmes MD, Malik VS, Willett WC, Hu FB: White rice, brown rice, and risk of type 2 diabetes in US men and women. Arch Intern Med 2010; 170:961-969.

30 Fung TT, Hu FB, Pereira MA, Liu S, Stampfer MJ, Colditz GA, Willett WC: Whole-grain intake and the risk of type 2 diabetes: a prospective study in men. Am J Clin Nutr 2002;76: 535-540.

31 Liu S, Manson JE, Stampfer MJ, Hu FB, Giovannucci E, Colditz GA, Hennekens $\mathrm{CH}$, Willett WC: A prospective study of wholegrain intake and risk of type 2 diabetes mellitus in US women. Am J Public Health 2000; 90:1409-1415.

32 Parker ED, Liu S, Van Horn L, Tinker LF, Shikany JM, Eaton CB, Margolis KL: The association of whole grain consumption with incident type 2 diabetes: the women's health initiative observational study. Ann Epidemiol 2013;23:321-327.

33 Schulze MB, Schulz M, Heidemann C, Schienkiewitz A, Hoffmann K, Boeing H: Fiber and magnesium intake and incidence of type $2 \mathrm{di}$ abetes: a prospective study and meta-analysis. Arch Intern Med 2007;167:956-965.

34 Du S, Lü B, Wang Z, Zhai F: [Transition of dietary pattern in China]. Wei Sheng Yan Jiu 2001;30:221-225.

35 Shay CM, Van Horn L, Stamler J, Dyer AR, Brown IJ, Chan Q, Miura K, Zhao L, Okuda N, Daviglus ML: Food and nutrient intakes and their associations with lower bmi in middle-aged us adults: the international study of macro-/micronutrients and blood pressure (INTERMAP). Am J Clin Nutr 2012;96:483491.

36 Yang L, Chen JH, Lv J, Wu Q, Xu T, Zhang H, Liu QH, Yang HK: Rice protein improves adiposity, body weight and reduces lipids level in rats through modification of triglyceride metabolism. Lipids Health Dis 2012;11: 24.

37 James SA, Pushpara T, Thavarajah D, Premakumara S, Abeysekara K, Sotheeswaran S: Rice (Oryza sativa L.) resistant starch and novel processing methods to increase resistant starch concentration. National Meeting \& Exposition of the American Chemical Society (ACS), 2015.

38 Radhika G, Van Dam RM, Sudha V, Ganesan A, Mohan V: Refined grain consumption and the metabolic syndrome in urban Asian Indians (Chennai urban rural epidemiology study 57). Metabolism 2009;58:675-681.

39 Liu S, Manson JE, Stampfer MJ, Holmes MD, $\mathrm{Hu}$ FB, Hankinson SE, Willett WC: Dietary glycemic load assessed by food-frequency questionnaire in relation to plasma high-density-lipoprotein cholesterol and fasting plasma triacylglycerols in postmenopausal women. Am J Clin Nutr 2001;73:560-566. 
40 Mancini M, Mattock M, Rabaya E, Chait A, Lewis B: Studies of the mechanisms of carbohydrate-induced lipaemia in normal man. Atherosclerosis 1973;17:445-454.

41 Parks EJ, Krauss RM, Christiansen MP, Neese RA, Hellerstein MK: Effects of a low-fat, highcarbohydrate diet on VLDL-triglyceride assembly, production, and clearance. J Clin Invest 1999;104:1087-1096.

42 Ma Y, Li Y, Chiriboga DE, Olendzki BC, Hebert JR, Li W, Leung K, Hafner AR, Ockene IS: Association between carbohydrate intake and serum lipids. J Am Coll Nutr 2006;25: 155-163.

43 Hauner H, Bechthold A, Boeing H, Brönstrup A, Buyken A, Leschik-Bonnet E, Linseisen J, Schulze M, Strohm D, Wolfram G: Evidence- based guideline of the German nutrition society: carbohydrate intake and prevention of nutrition-related diseases. Ann Nutr Metab 2012;60(suppl 1):1-58.

44 Reynolds K, Gu D, Whelton PK, Wu X, Duan $\mathrm{X}, \mathrm{Mo} \mathrm{J}, \mathrm{He} \mathrm{J}$ : Prevalence and risk factors of overweight and obesity in China. Obesity (Silver Spring) 2007;15:10-18

45 Wang ZW, Wang X, Li X, Chen Z, Zhao LC, Li Y, Wu YF: [Prevalence and trend of metabolic syndrome in middle-aged Chinese population]. Zhonghua Liu Xing Bing Xue Za Zhi 2009;30:596-600.

46 DIAMOND Project Group: Incidence and trends of childhood type 1 diabetes worldwide 1990-1999. Diabet Med 2006;23:857866.
47 Zhang G, Malik VS, Pan A, Kumar S, Holmes MD, Spiegelman D, Lin X, Hu FB: Substituting brown rice for white rice to lower diabetes risk: a focus-group study in Chinese adults. J Am Diet Assoc 2010;110:1216-1221.

48 Zhang G, Pan A, Zong G, Yu Z, Wu H, Chen X, Tang L, Feng Y, Zhou H, Chen X, Li H, Hong B, Malik VS, Willett WC, Spiegelman D, Hu $\mathrm{FB}$, Lin X: Substituting white rice with brown rice for 16 weeks does not substantially affect metabolic risk factors in middle-aged Chinese men and women with diabetes or a high risk for diabetes. J Nutr 2011;141:1685-1690.

49 Jones-Smith JC, Popkin BM: Understanding community context and adult health changes in China: development of an urbanicity scale. Soc Sci Med 2010;71:1436-1446. 